\title{
Cancer-associated peritoneal mesothelial cells lead the formation of pancreatic cancer peritoneal dissemination
}

\author{
TOSHIYA ABE ${ }^{1}$, KENOKI OHUCHIDA $^{1}$, KAZUHIRO KOIKAWA ${ }^{1}$, SHO ENDO $^{1}$, TAKASHI OKUMURA ${ }^{1}$, \\ MASAFUMI SADA ${ }^{1}$, KOHEI HORIOKA ${ }^{1}$, BIAO ZHENG ${ }^{1}$, TAIKI MORIYAMA ${ }^{1}$, KOHEI NAKATA ${ }^{1}$, \\ YOSHIHIRO MIYASAKA ${ }^{1}$, TATSUYA MANABE ${ }^{1}$, TAKAO OHTSUKA ${ }^{1}$, EISHI NAGAI ${ }^{1}$, \\ KAZUHIRO MIZUMOTO ${ }^{1}$, MAKOTO HASHIZUME ${ }^{2}$ and MASAFUMI NAKAMURA ${ }^{1}$ \\ Departments of ${ }^{1}$ Surgery and Oncology and ${ }^{2}$ Advanced Medical Initiatives, \\ Graduate School of Medical Sciences, Kyushu University, Fukuoka 812-8582, Japan
}

Received September 26, 2016; Accepted December 21, 2016

DOI: $10.3892 /$ ijo.2016.3829

\begin{abstract}
The interaction between the cancer cells and the peritoneal mesothelial cells (PMCs) plays an important role in the peritoneal dissemination in several types of cancer. However, the role of PMCs in the peritoneal dissemination of pancreatic cancer remains unclear. In the present study, we investigated the interaction between the pancreatic cancer cells (PCCs) and the PMCs in the formation of peritoneal dissemination in vitro and in vivo. The tumor-stromal interaction of PCCs and PMCs significantly enhanced their mobility and invasiveness and enhanced the proliferation and anoikis resistance of PCCs. In a 3D organotypic culture model of peritoneal dissemination, co-culture of PCCs and PMCs significantly increased the cells invading into the collagen gel layer compared with mono-culture of PCCs. PMCs pre-invaded into the collagen gel, remodeled collagen fibers, and increased parallel fiber orientation along the direction of cell invasion. In the tissues of peritoneal dissemination of the KPC (LSL-Kras ${ }^{\mathrm{G} 12 \mathrm{D} /+}$;
\end{abstract}

Correspondence to: Dr Kenoki Ohuchida and Dr Masafumi Nakamura, Department of Surgery and Oncology, Graduate School of Medical Sciences, Kyushu University, 3-1-1 Maidashi, Fukuoka 812-8582, Japan

E-mail: kenoki@surg1.med.kyushu-u.ac.jp

E-mail:mnaka@surg1.med.kyushu-u.ac.jp

Abbreviations: PMCs, peritoneal mesothelial cells; PCCs, pancreatic cancer cells; KPC, (LSL-Kras ${ }^{\mathrm{G} 12 \mathrm{D} /++} ; \mathrm{LSL}-\mathrm{Trp} 53$ ${ }^{\mathrm{R} 172 \mathrm{H} /+}$;Pdx-1-Cre) mouse model; MMT, mesothelial-to-mesenchymal transition; CAFs, cancer associated fibroblasts; DMEM, Dulbecco's modified Eagle's medium; FBS, fetal bovine serum; TGF- $\beta 1$, transforming growth factor- $\beta 1$; aPMCs, activated PMCs; H\&E, hematoxylin and eosin; $\alpha$-SMA, $\alpha$-smooth muscle actin; CM, conditioned medium; PBS, phosphate-buffered saline; FN1, fibronectin 1; qRT-PCR, real-time quantitative reverse transcription polymerase chain reaction; PSCs, pancreatic stellate cells

Key words: peritoneal mesothelial cells, pancreatic cancer, peritoneal dissemination, tumor-stromal interaction, remodeling
LSL-Trp53 $\left.{ }^{\mathrm{R} 172 \mathrm{H} /+} ; \mathrm{Pdx}-1-\mathrm{Cre}\right)$ transgenic mouse, the monolayer of PMCs was preserved in tumor-free areas, whereas PMCs around the invasive front of peritoneal dissemination proliferated and invaded into the muscle layer. In vivo, intraperitoneal injection of PCCs with PMCs significantly promoted peritoneal dissemination compared with PCCs alone. The present data suggest that the cancer-associated PMCs have important promoting roles in the peritoneal dissemination of PCCs. Therapy targeting cancer-associated PMCs may improve the prognosis of patients with pancreatic cancer.

\section{Introduction}

Pancreatic cancer is the fourth leading cause of cancer-related death in the United States, with a 5-year overall survival rate of 3-5\% (1). Most pancreatic cancer patients have distant metastasis at diagnosis, and the prognosis of patients with peritoneal dissemination is extremely poor $(2,3)$. Furthermore, peritoneal dissemination induces bowel obstruction and formation of malignant ascites, which leads to poor performance status (3). Therefore, it is important to determine a mechanism to control the peritoneal dissemination of pancreatic cancer.

Pancreatic cancer is characterized by excessive desmoplasia, which plays a crucial role in its aggressive behavior through the tumor-stroma interaction (4). In the process of peritoneal dissemination, cancer cells detached from the primary tumor are transported by peritoneal fluid and disseminate in the peritoneum (5). The peritoneum consists of a monolayer of peritoneal mesothelial cells (PMCs) supported by a basement membrane that rest on a layer of connective tissue with fibroblasts, mast cells, macrophages and vessels (6). Therefore, the tumor-stroma interaction is important in the process of peritoneal dissemination (7). We recently investigated the tumor-stroma interaction in peritoneal dissemination in pancreatic cancer and found that peritoneal myofibroblasts contributed to the promotion of peritoneal dissemination in pancreatic cancer (8). Previous studies reported that myofibroblasts were derived from resident peritoneal fibroblasts, bone marrow progenitor cells, the primary tumor itself or PMCs (9-12). However, the origin of peritoneal myofibroblasts in pancreatic cancer is not yet clearly understood. 
PMCs generally act as a passive barrier and play important roles in the response to wound healing and infection (13). Other reports also showed that PMCs are converted into myofibroblasts via mesothelial-to-mesenchymal transition (MMT) by peritoneal dialysis (9) and in ovarian and gastric cancers $(14,15)$. PMCs that are converted into myofibroblasts via MMT affect cancer cells as cancer associated fibroblasts $(\mathrm{CAFs})$ in ovarian and gastric cancers $(14,15)$. CAFs support the malignant progression of tumors by promoting growth, survival, angiogenesis, inflammation, drug resistance and invasion and metastasis of tumors (16). A previous study showed that heat-shock-factor-1 was one of the potent activator of CAFs promoting malignancy (17). On the other hand, the interaction between PMCs and cancer cells promoted the proliferation and invasiveness of cancer cells in ovarian $(14,18,19)$ and gastric $(10,20)$ cancer. Moreover, adhesive interaction between cancer cells and PMCs played an important role in peritoneal dissemination (21). A recent study revealed molecular mechanisms of peritoneal dissemination in gastric cancer (22). However, the role of PMCs in the peritoneal dissemination of pancreatic cancer remains unclear.

In the present study, we revealed the interaction between the pancreatic cancer cells (PCCs) and PMCs in the process of peritoneal dissemination in vitro and in vivo.

\section{Materials and methods}

Cell isolation and culture conditions. PMCs were isolated from ascites recovered from patients with no evidence of distant metastasis who underwent curative resection for pancreatic cancer, as previously described (9,23-25). Briefly, after ascites were centrifuged at $1,500 \mathrm{rpm}$ for $10 \mathrm{~min}$, the cell pellets were resuspended in Dulbecco's modified Eagle's medium (DMEM) supplemented with 10\% fetal bovine serum (FBS), penicillin and streptomycin and cultured in Collagen type I coated dishes (Iwaki, Co., Ltd., Tokyo, Japan). The isolated cells were identified as PMCs by their polygonal morphology and the expression of calretinin and cells between passages 2 and 5 were used for assays. We confirmed that there was no contamination with fibroblasts, endothelial cells or malignant cells. PMCs were activated by transforming growth factor- $\beta 1$ (TGF- $\beta 1$ ) administration and these cells were regarded as activated PMCs (aPMCs), as previously described $(9,10)$. Briefly, PMCs were incubated with or without $10 \mathrm{ng} / \mathrm{ml}$ TGF- $\beta 1$ (R\&D Systems, Oxon, UK) in DMEM with 1\% FBS for $48 \mathrm{~h}$. We also used the following three PCC lines: SUIT-2 (Health Science Research Bank, Osaka, Japan), AsPC-1 (American Type Culture Collection, Manassas, VA, USA) and PANC-1 (RIKEN BioResource Center, Tsukuba, Japan). These cells were maintained as previously described (26).

Establishment of immortalized PMCs. We cloned the DNA encoding hTERT and SV40 Large T in the pLVSIN vector. We used these vectors to construct lentiviral particles for infection of PMCs, followed by G418 selection.

Stable luciferase-expressing SUIT-2 cells. The firefly luciferase (GenTarget, \#LVP326) expression vector was transfected into SUIT-2 cells according to the manufacturer's instructions. Blastidine S hydrochloride (\#15205; Sigma-Aldrich) was used for selection for more than 3 weeks. Luciferase expression was confirmed by a significant increase in emission after adding $150 \mu \mathrm{g} / \mathrm{ml}$ D-Luciferin potassium salt (\#LK10000; OZ Biosciences, Marseille, France) as compared with wild-type SUIT-2 cells.

Immunohistochemical analysis. Tissues of peritoneal dissemination of pancreatic cancer in KPC (LSL-Kras G12D/+; LSL-Trp53 $\left.3^{\mathrm{R} 172 \mathrm{H} /+} ; \mathrm{Pdx}-1-\mathrm{Cre}\right)$ transgenic mice (27) were evaluated by hematoxylin and eosin (H\&E), CK19, calretinin and $\alpha$-smooth muscle actin ( $\alpha$-SMA) immunohistochemical staining. Tissues were sliced to a thickness of $4 \mu \mathrm{m}$ and incubated with rabbit anti-CK19 antibody (\#133496, 1:100; Abcam), mouse anti-calretinin antibody (\#M7245, 1:25; Dako) or mouse anti- $\alpha$-SMA antibody (\#M0851, 1:100; Dako) overnight at $4^{\circ} \mathrm{C}$. The staining was performed using serial sections.

Conditioned medium of PMCs. To obtain conditioned medium of PMCs (PMCs-CM), PMCs were seeded and cultured until subconfluent. The medium was then replaced with DMEM serum-free medium and the supernatants were collected after $48 \mathrm{~h}$ of incubation.

Matrigel invasion and migration assays. The invasiveness and migration capacities of PCCs and PMCs were assessed by determining the number of cells that invaded or migrated across Transwell chambers, as previously described (26). For co-cultures, PMCs $\left(4.0 \times 10^{4} /\right.$ well) or aPMCs $\left(4.0 \times 10^{4} /\right.$ well $)$ in $750 \mu 1$ of DMEM supplemented with $10 \%$ FBS or medium alone were seeded in the lower chambers for $24 \mathrm{~h}$, and PCCs (4.0x10 $10^{4}$ well) in $250 \mu \mathrm{l}$ of DMEM supplemented with $10 \%$ FBS were placed in the upper Transwell chamber $(8 \mu \mathrm{m}$ pore size; Becton-Dickinson, Franklin Lakes, NJ, USA) containing $100 \mathrm{ml}$ of reconstituted Matrigel-coated membrane (20 mg/well; BD Biosciences, Bedford, MA, USA). After incubation for $48 \mathrm{~h}$, cell invasion was evaluated by counting the number of cells that invaded through the Transwell chambers. Cell migration was assessed after incubation for $24 \mathrm{~h}$ using uncoated Transwell chambers. To assess the invasiveness of PMCs, each lower well was seeded with PCCs (4.0x 104/well) in $750 \mu \mathrm{l}$ of DMEM supplemented with $10 \%$ FBS or medium alone and incubated for $24 \mathrm{~h}$. PMCs $\left(4.0 \times 10^{4} /\right.$ well) in $250 \mu \mathrm{l}$ of DMEM supplemented with 10\% FBS were seeded in each upper well and incubated for $24 \mathrm{~h}$ for the invasion assay and $12 \mathrm{~h}$ for the migration assay. In both assays and at each timepoint, invading or migrated cells at the bottom of the chamber were fixed with $70 \%$ ethanol and stained with $\mathrm{H} \& \mathrm{E}$, and five random fields at $\mathrm{x} 200$ magnification were counted under a light microscope. Each experiment was performed in triplicate and repeated at least three times.

Adhesion assay. The adhesion ability of PCCs was determined as previously described (28). Briefly, PMCs and aPMCs $\left(8.0 \times 10^{4} /\right.$ well) were cultured in a monolayer in 96-well Collagen I coated plates overnight. Collagen I was used as the principal extracellular matrix molecule. PCCs were labeled with CellTracker ${ }^{\mathrm{TM}}$ Green CMFDA (Life Technologies, Eugene OR, USA). PCCs $\left(4.0 \times 10^{4} /\right.$ well $)$ were added to the 96-well Collagen I coated plates containing confluent PMCs 
or aPMCs or without cells, and cells were incubated for $3 \mathrm{~h}$ at $37^{\circ} \mathrm{C}$. The plates were then washed three times with $200 \mu \mathrm{l}$ of phosphate-buffered saline (PBS) to remove the non-adherent tumor cells. The number of adhered PCCs was determined in five random fields at $\mathrm{x} 200$ magnification using a fluorescent microscope (BZ-9000; Keyence Corp., Osaka, Japan). Each experiment was performed in triplicate and repeated at least three times.

Cell viability assay (adhered and non-adhered conditions). PCCs (1.0x10 $3 /$ well) were seeded (Greiner Bio-One GmbH, Frickenhausen, Germany) and cell viability examined using the CellTiter-Glo ${ }^{\circledR}$ Luminescent Cell Viability assay kit (G7570; Promega, Madison, WI, USA) after culture for 24, 48 and $72 \mathrm{~h}$ following the manufacturer's instructions. PCCs $\left(1.0 \times 10^{3} /\right.$ well $)$ were incubated with or without PMC-CM in $10 \% \mathrm{FBS} / \mathrm{DMEM}$ in 96-well plates. In non-adhered conditions, PCCs (1.0x10 $3 /$ well) were cultured in a 96-well ultra-low adherence plate (Corning Costar CLS7007). Background was subtracted using values from wells containing only culture medium. Each experiment was performed in triplicate and repeated at least three times.

Apoptosis assay. PCCs (1.0x10\% /well) were incubated with or without PMCs-CM in DMEM serum-free medium in a $90 \mathrm{~mm}$ ultra-low adherence plate (Nunclon Sphera Dishes; Thermo Fisher Scientific) for $72 \mathrm{~h}$ and the cells were lysed and subjected to western blotting to determine the expression of apoptosis regulators.

$3 D$ organotypic culture model. To assess the process of invasiveness of PCCs and PMCs, the 3D organotypic culture model was set up as previously described (20) with minor modifications. Briefly, $1000 \mu \mathrm{l}$ of the gel containing $2 \mathrm{mg} / \mathrm{ml}$ Collagen I (BD Biosciences) and $2.5 \mathrm{mg} / \mathrm{ml}$ Matrigel was laid onto the upper Transwell chambers (6-well). PMCs $\left(6.0 \times 10^{5} /\right.$ well) were added on the gels in DMEM containing 1\% FBS. After $6 \mathrm{~h}$, PCCs $\left(6.0 \times 10^{5} /\right.$ well $)$ were added on the monolayer of PMCs. The bottom well was filled with DMEM containing $10 \%$ FBS. SUIT-2 cells were labeled with CellTracker ${ }^{\mathrm{TM}}$ Green and PMCs were labeled with CellTracker ${ }^{\mathrm{TM}}$ Red CMTPX (Life Technologies). A laser-scanning confocal fluorescence microscope (A1R; Nikon) was used for immunofluorescence microphotography. After incubation for 10 days, the gels were fixed in $4 \%$ paraformaldehyde and sections were cut into $4 \mu \mathrm{m}$ sections for H\&E staining and incubated with rabbit Ki-67 antibody (\#16667, 1:100; Abcam) or rabbit anti-CEA (\#RB-368-A, 1:250; Thermo Fisher Scientific) overnight at $4^{\circ} \mathrm{C}$. The Ki-67 labeling index was calculated by dividing the number of $\mathrm{Ki}-67$ positive nuclei by the total number of nuclei in five random fields at x200 magnification.

Analysis of collagen fiber orientation. The orientation of the 3D gel collagen fibers was analyzed using OrientationJ (an ImageJ-plug-in) (v.1.48u; National Institute of Health, Bethesda, MD, USA) (29). Counts of the total fibers as well as each orientation angle were measured, and the angles were determined by approximating the relative angle every $10^{\circ}$. To allow for comparison of acquired images, we set the direction of the invading cells to $0^{\circ}$ in the invading area and the tangential direction of the initial cell cluster surface to $0^{\circ}$ in the non-invading area.

Real-time quantitative reverse transcription polymerase chain reaction ( $q R T-P C R$ ). qRT-PCR was performed using the iTaq Universal SYBR-Green One-Step kit and CFX96 Touch Real-Time PCR Detection systems (Bio-Rad Laboratories). The primers were purchased from Takara Bio (Kusatsu, Japan). Human GAPDH gene was used as the endogenous control gene. The following primers were used in the present study: E-cadherin, forward, 5'-AAGTGCTGCAGCCAAAGAC AGA-3' and reverse, 5'-AAATTGCCAGGCTCAATGACA AG-3'; fibronectin 1 (FN1), forward, 5'-ACAGAACTATG ATGCCGACCAGAAG-3' and reverse, 5'-ACTGATCTCCA ATGCGGTACATGA-3'; GAPDH, forward, 5'-GCACCGT CAAGGCTGAGAAC-3' and reverse, 5'-TGGTGAAGACG CCAGTGGA-3'.

Western blot analysis. Whole cell lysates were prepared in PRO-PREP solution (iNtRON Biotechnology, Seongnam, Korea) from PMCs, aPMCs and PCCs. Proteins from cell lysates were fractioned by SDS-PAGE and transferred to a polyvinylidene difluoride membrane (Bio-Rad Laboratories). The membrane was incubated overnight at $4^{\circ} \mathrm{C}$ with the following antibodies: anti-E-cadherin (\#3195, 1:1,000; Cell Signaling Technology, Danvers, MA, USA), anti-vimentin (\#5741, 1:1,000; Cell Signaling Technology), anti- $\alpha$-SMA (\#M0851, 1:500; Dako), anti-FN1 (\#sc-9068, 1:200; Santa Cruz Biotechnology), anti-calretinin (\#92341, 1:1,000; Abcam), anti-CEA (\#RB-368-A, 1:200; Thermo Fisher Scientific), anti-cleaved caspase-3 (\#9661, 1:1,000; Cell Signaling Technology) and anti- $\beta$-actin (\#8227, 1:5,000; Abcam). The membrane was then probed with horseradish-peroxidaseconjugated secondary antibodies (Cell Signaling Technology). Immunoblots were detected by enhanced chemiluminescence.

Animal experiments. All experiments with mice were conducted and approved by the Ethics Committee of Kyushu University. The model of intraperitoneal injection of BALB/c nu/nu mice (5-6 weeks of age; Kyudo Co.) was used to analyze the dissemination activity of PCCs alone and PCCs with PMCs. All animals were bred in laminar-flow cabinets under specific pathogen-free conditions. The mice were anesthetized with ether and suspensions of luciferase-expressing SUIT-2 cells $\left(2 \times 10^{5}\right)$ alone in $200 \mu \mathrm{l}$ PBS or with PMCs $\left(1 \times 10^{6}\right)$ in $200 \mu \mathrm{l}$ PBS were injected into the peritoneal cavity of groups of 6 mice. Luciferin luminescence was measured at 21 days after intraperitoneal injection using IVIS Spectrum (Caliper Life Sciences, Waltham, MA, USA) after injecting $150 \mathrm{mg}$ D-Luciferin (\#LK10000; OZ Biosciences) into the intraperitoneal cavity of anesthetized mice. Luminescence was quantified using Living Image software version 4.4 (Summit Pharmaceuticals International Corp., Tokyo, Japan). All mice were sacrificed at 21 days after the evaluation of luciferin luminescence and the disseminated nodules $>3 \mathrm{~mm}$ in size were counted.

Statistical analysis. Results are presented as means \pm SD. Comparisons between the two groups were conducted using the Student's t-test. Tumor volume and disseminated nodules in vivo 


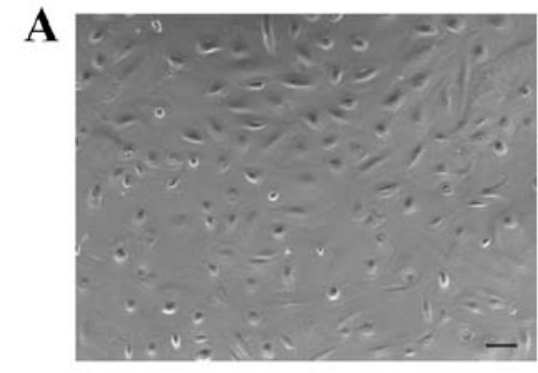

PMCs

\section{B}

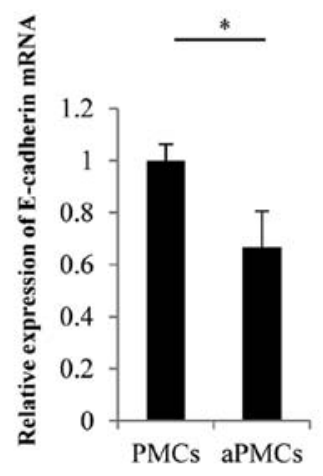

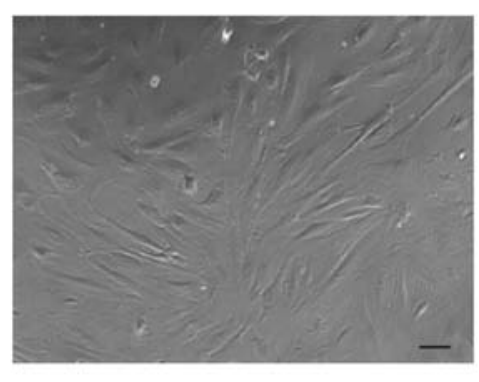

aPMCs
C

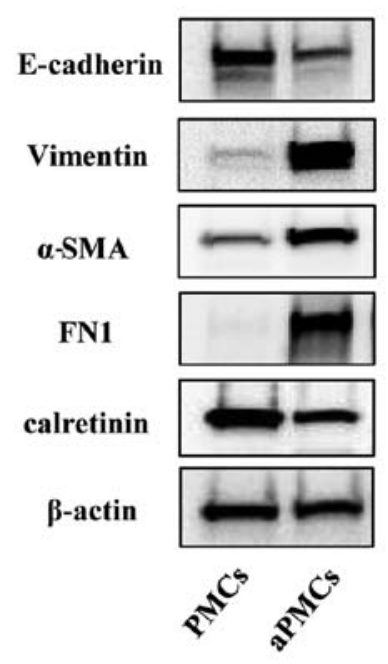

Figure 1. TGF- $\beta 1$ induces mesothelial-to-mesenchymal transition (MMT) in PMCs. (A) Representative microphotographs of PMCs and activated PMCs (aPMCs) that were incubated with TGF- $\beta 1(10 \mathrm{ng} / \mathrm{ml})$ for $48 \mathrm{~h}$. Original magnification, x100. Scale bars, $100 \mu \mathrm{m}$. (B) qRT-PCR analysis and (C) western blot analysis of PMCs and aPMCs for the indicated molecular factors. ${ }^{*} \mathrm{P}<0.05 ;{ }^{* * *} \mathrm{P}<0.001$.

were analyzed using the Wilcoxon test. Statistical significance was defined as $\mathrm{P}<0.05$. All statistical analyses were performed using JMP 11 software (SAS Institute, Cary, NC, USA).

\section{Results}

Verification of PMCs and aPMCs. The isolated cells were identified as PMCs by their polygonal morphology and the expression of calretinin (Fig. 1A and C). Representative microphotographs of aPMCs showed spindle-like morphology compared with PMCs (Fig. 1A). To verify the MMT of PMCs, the expressions of MMT markers were analyzed by qRT-PCR and western blotting. The gene and protein expression of E-cadherin was decreased in PMCs, whereas the expression levels of $\alpha$-SMA, FN1 and vimentin were increased (Fig. 1B and C) confirming MMT of PMCs.

The tumor-stromal interaction of PCCs and PMCs significantly enhances their migration and invasiveness in indirect co-culture. To investigate the tumor-stromal interaction between PMCs and PCCs, we evaluated the effect of PMCs on PCC migration and invasiveness using indirect co-culture. We found that PMCs significantly enhanced the migration (Fig. 2A) and the invasiveness (Fig. 2B) of PCCs in indirect co-culture (both $\mathrm{P}<0.001$ ). Furthermore, co-culture with aPMCs significantly enhanced the migration (Fig. 2A) and invasiveness (Fig. 2B) of PCCs compared with the co-culture with PMCs (both $\mathrm{P}<0.001$ ). We also investigated the effect of co-cultured PCCs on the mobility and invasiveness of PMCs and found that PCCs significantly enhanced the migration
(Fig. 2C) and the invasiveness (Fig. 2D) of PMCs (both $\mathrm{P}<0.001)$.

Adhesion ability of PCCs to PMCs, aPMCs or Collagen I. Next, we assessed the adhesion ability of PCCs to PMCs, aPMCs or Collagen I and found that the adhesion ability of PCCs to PMCs was significantly decreased compared with the adhesion to Collagen I, whereas the adhesion ability of PCCs to aPMCs was significantly increased compared with the adhesion to PMCs (Fig. 3; $\mathrm{P}<0.001$ ).

PMCs enhance proliferation and anoikis resistance of PCCs. We found that PMCs significantly enhanced the proliferation of PCCs compared with controls both in adhered (Fig. 4A; $\mathrm{P}<0.001$ ) and non-adhered conditions (Fig. 4B; SUIT-2 cells, $\mathrm{P}<0.05$; AsPC-1 cells; $\mathrm{P}<0.001)$. Furthermore, addition of PMCs-CM decreased the expression of cleaved caspase- 3 of PCCs compared with controls (Fig. 4C).

PMCs enhance invasiveness and proliferation of PCCs and remodel collagen fibers in the $3 D$ organotypic culture model. We evaluated the invasion process of PCCs and PMCs using a 3D organotypic culture model and confocal fluorescence microscopy. We found that the invasion distance of PMCs was significantly longer than that of SUIT-2 cells (Fig. 5A and B; $\mathrm{P}<0.001)$. H\&E sections showed that the co-culture of SUIT-2 cells and PMCs showed a significant increase in the number of cells invading into the collagen gel layer compared with mono-culture of SUIT- 2 cells, which did not invade into the collagen gel layer (Fig. 5C and D; $\mathrm{P}<0.001$ ). To distinguish 
A
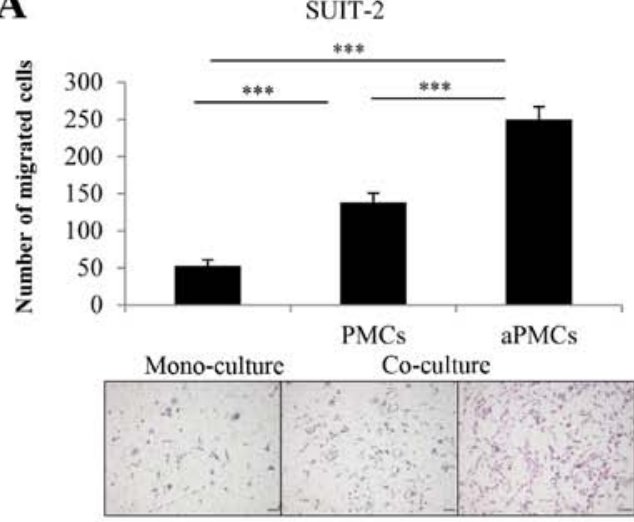

B

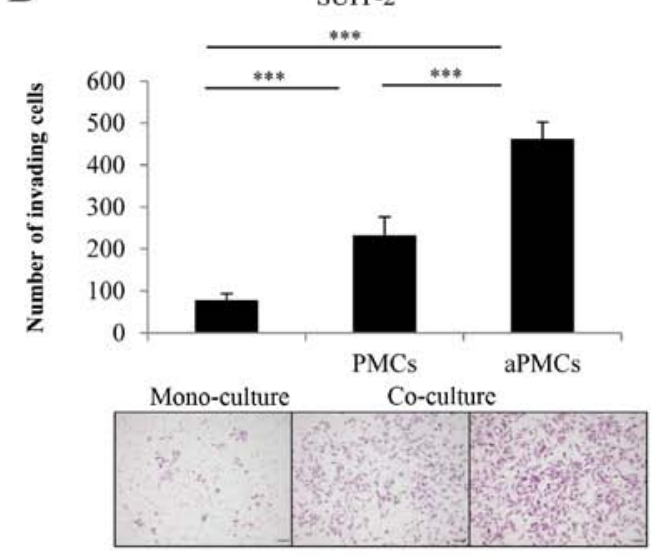

C

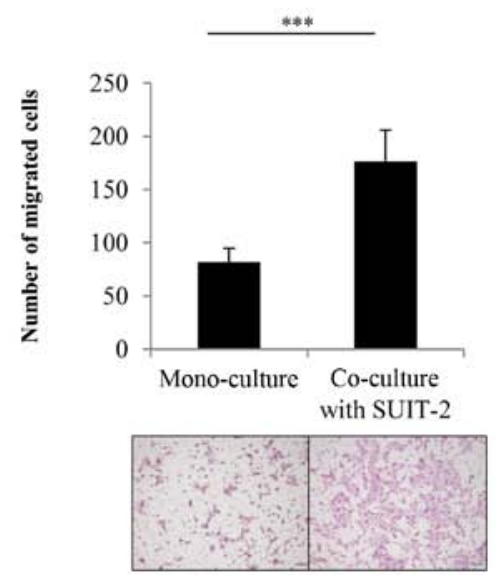

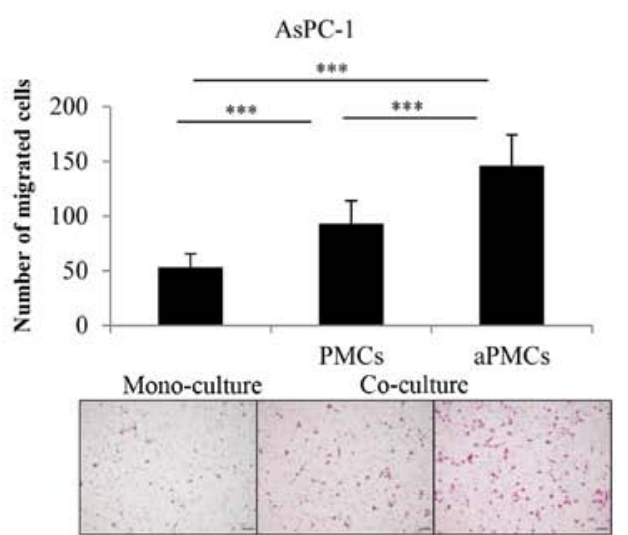

AsPC-1

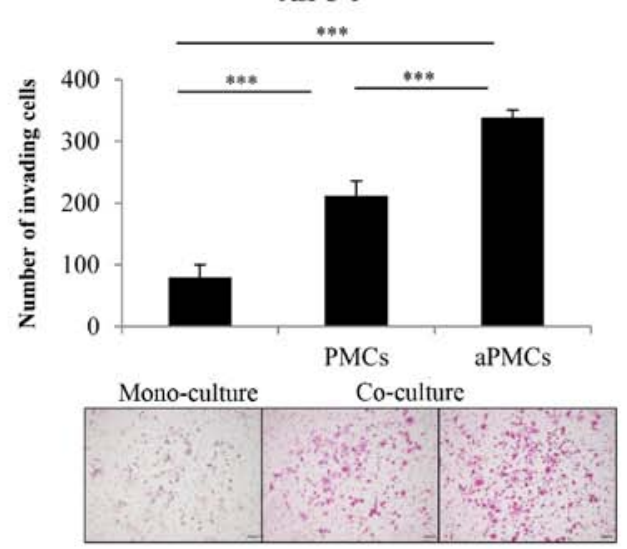

D
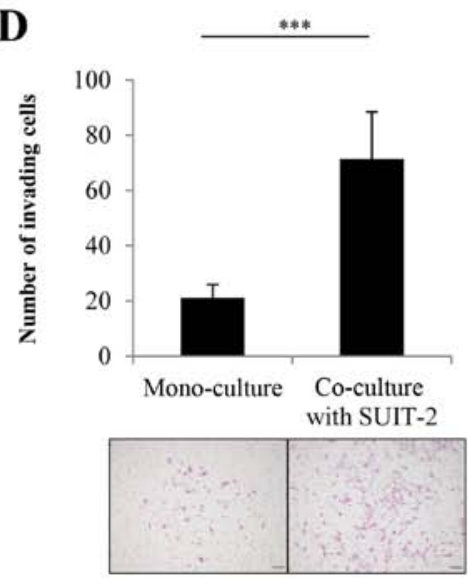

Figure 2. The tumor-stromal interaction of PCCs and PMCs significantly promoted their migration and invasiveness in indirect co-culture. (A) Cell migration assays and (B) cell invasion assays of PCCs alone (first column) and PCCs co-cultured with PMCs (second column) or aPMCs (third column). The images show representative H\&E stainings. (C and D) The migration and invasiveness of PMCs were enhanced when co-cultured with PCCs. H\&E staining; original magnification, $\mathrm{x} 100 .{ }^{* * *} \mathrm{P}<0.001$.

between PCCs and PMCs, the expression of CEA was assessed by western blotting and was positive only in SUIT-2 cells (Fig. 5E). Immunohistochemical staining showed that SUIT-2 cells expressing CEA invaded into the collagen gel layer upon co-culture with PMCs (Fig. 5F). Immunohistochemical staining of Ki-67 showed that co-culture of SUIT-2 cells and PMCs significantly enhanced proliferation compared with monoculture of SUIT-2 cells (Fig. 5G and H; P<0.001). To investigate the mechanism of PMC-induced invasiveness of PCCs, we focused on the collagen fiber orientation. We found that the collagen fibers in the co-cultures of SUIT-2 cells and
PMCs or the monocultures of PMCs displayed an organized parallel orientation along the cells invading into collagen gel layer compared with the random fiber arrangement detected in the monocultures of SUIT-2 cells (Fig. 5I and J).

PMCs exist in the invasive front of peritoneal dissemination of pancreatic cancer derived from KPC mice. To assess the role of $\mathrm{PMC}$ in peritoneal dissemination of pancreatic cancer in the KPC mouse, we focused on the invasive front of peritoneal dissemination. We performed H\&E staining and immunohistochemical staining of CK19 as a PCC marker, 

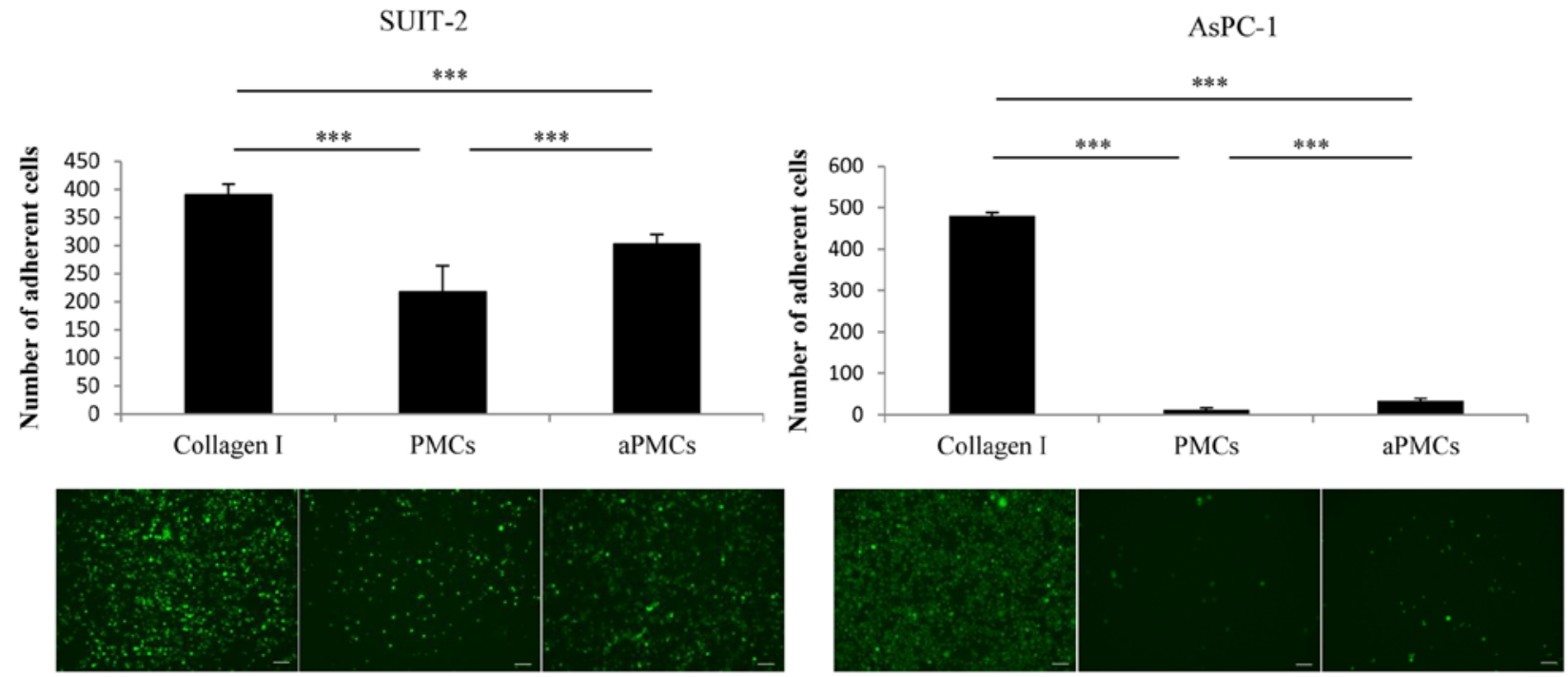

Figure 3. Adhesion ability of PCCs to collagen I, PMCs and aPMCs. PCCs were examined for adhesion ability with collagen I, PMCs or aPMCs. The top panel shows quantification of cell numbers. The bottom panel shows images by fluorescent microscope. Original magnification, $\mathrm{x} 100 ;{ }^{* * *} \mathrm{P}<0.001$.

A

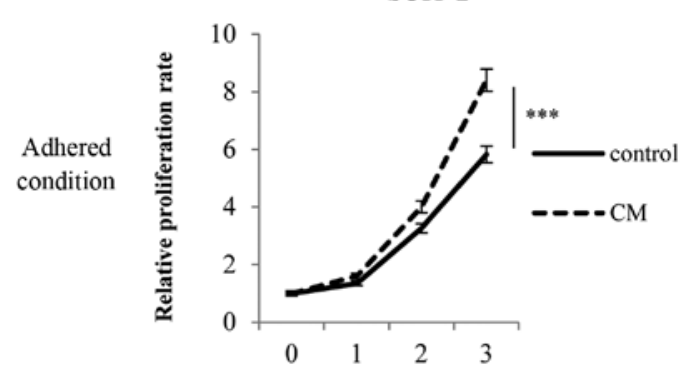

B

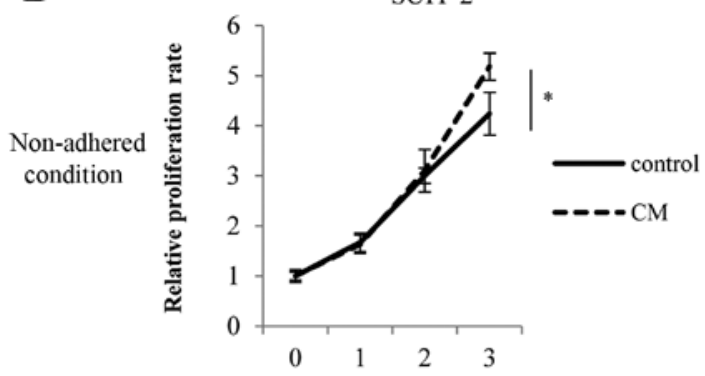

AsPC-1

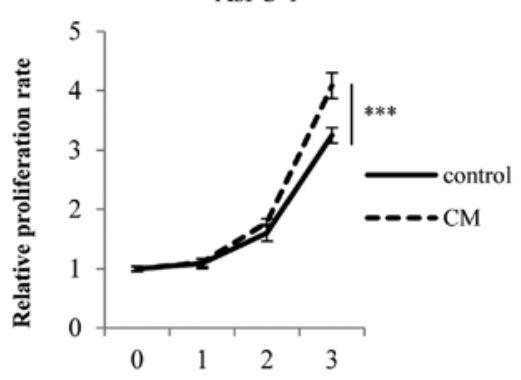

AsPC-1

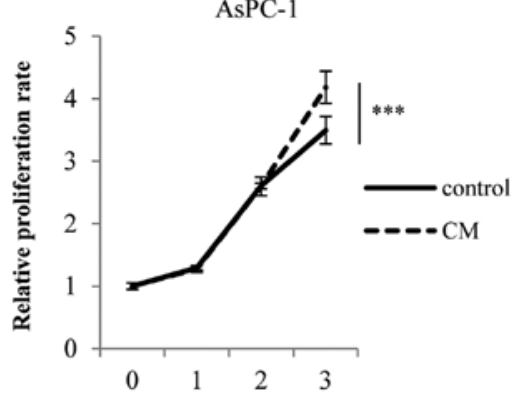

C

Cleaved caspase-3

$\beta$-actin

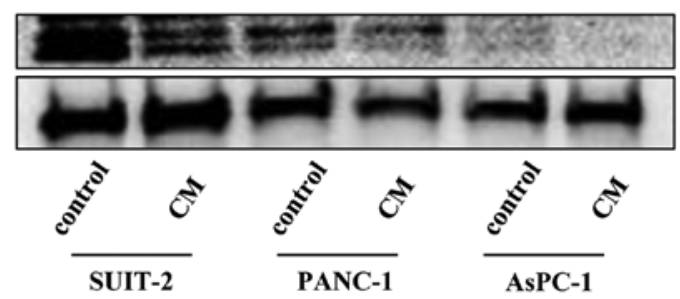

Figure 4. PMCs enhance proliferation and anoikis resistance of PCCs. PCCs were incubated with or without PMC-CM in (A) adhered conditions (96-well plates) or (B) non-adhered conditions (96-well ultra-low adherence plate) and cell viability was evaluated. (C) Western blot analysis of cleaved caspase-3 of PCCs in non-adhered conditions and in the presence of PMC-CM. ${ }^{*} \mathrm{P}<0.05,{ }^{* * *} \mathrm{P}<0.001$.

calretinin as a PMC marker and $\alpha$-SMA as a myofibroblast marker. The monolayer of PMCs was preserved in tumor-free areas, whereas in micro-peritoneal dissemination, multiple layers of PMCs were observed and a subset of PMCs invaded into the muscle layer (Fig. 6A). In macro-peritoneal dissemination, PMCs existed in the invasive front and pre-invaded into the muscle layer, whereas $\alpha$-SMA-positive and calretininnegative stromal cells existed in the tumor (Fig. 6B). 
A

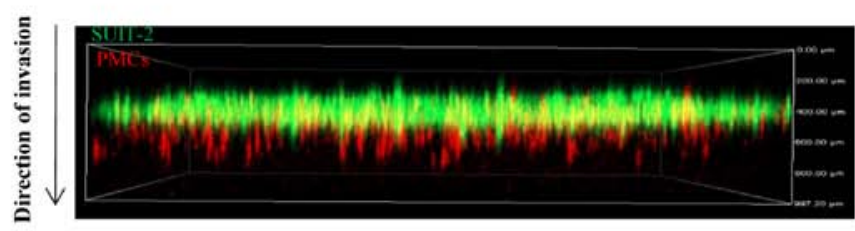

C

C

$\stackrel{+}{\mathrm{PMCs}}$

SUIT-2
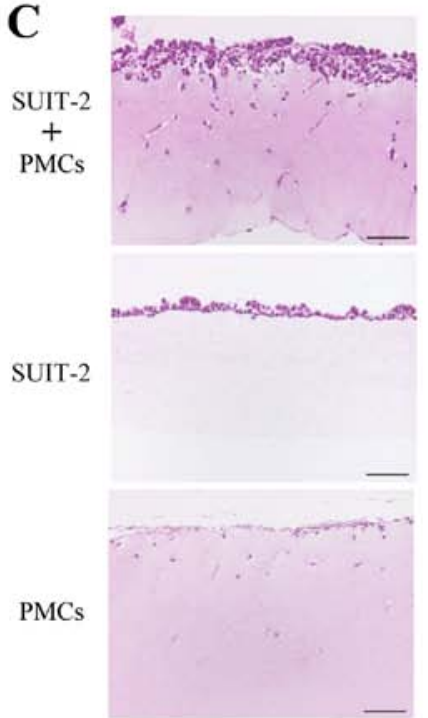

D

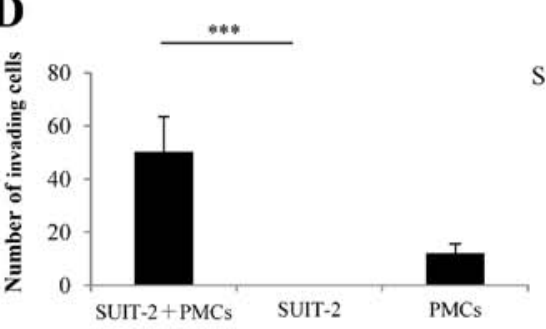

I
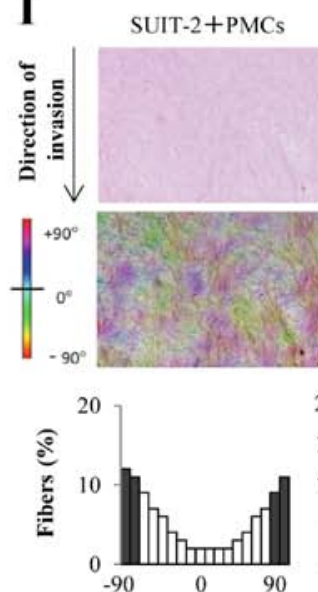

$\mathbf{E}$

CEA

$\beta$-actin
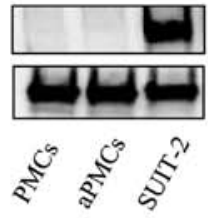

B

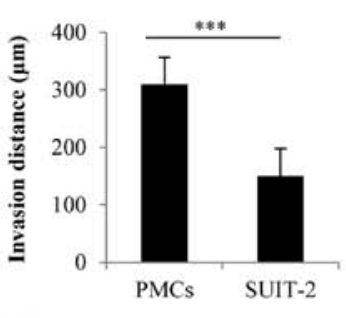

$\mathbf{F}$

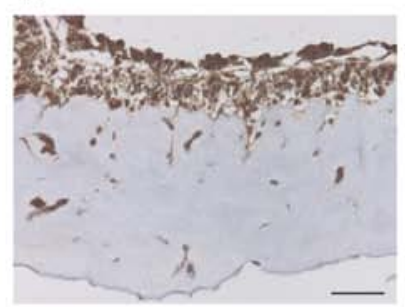

\section{G}

SUIT-2

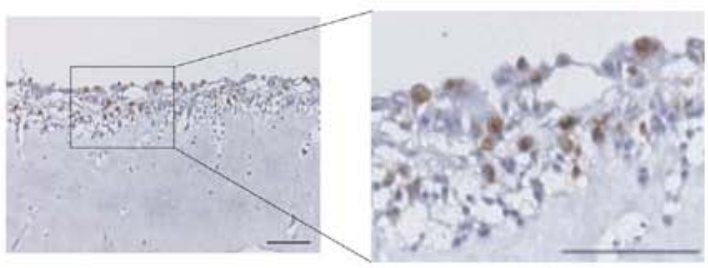

PMCs

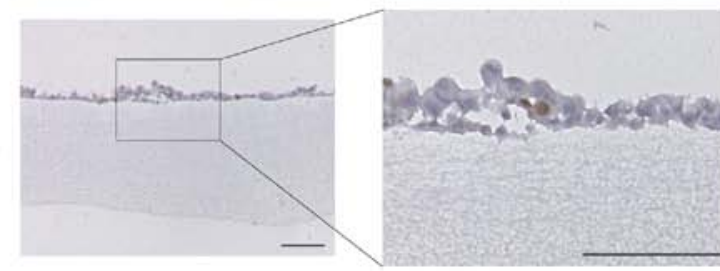

H
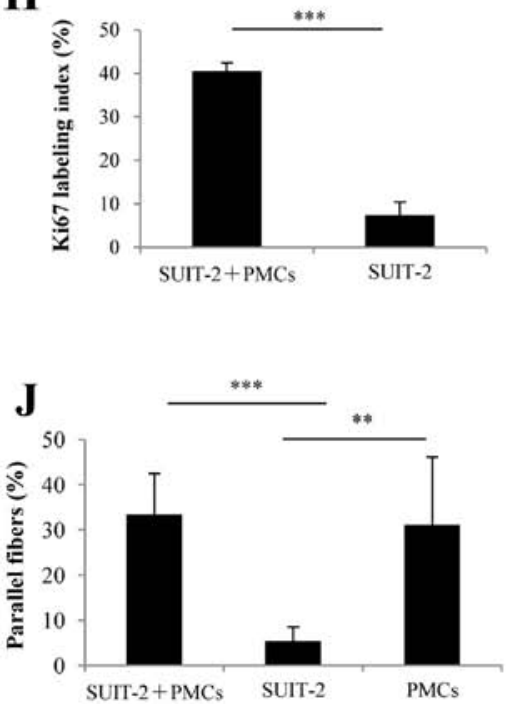

Figure 5. PMCs enhance invasiveness and proliferation of PCCs and remodel collagen fibers in the 3D organotypic culture model. (A) Representative confocal fluorescence images on day 5 showed that PMCs (red) pre-invaded into the collagen compared with SUIT-2 cells (green). (B) Quantification analysis of invasion distance of PMCs and SUIT-2 cells. (C) Representative image of H\&E sections of collagen gel on day 10 and (D) quantification analysis of the number of invaded cells. (E) Western blot analysis of CEA in PMCs, aPMCs and SUIT-2 cells. (F) Representative images of immunohistochemical staining showed that SUIT-2 cells expressing CEA invaded into the collagen gel layer when co-cultured with PMCs. (G) Representative images of immunohistochemical staining of Ki-67 and (H) Ki-67 labeling index showed that co-culture with SUIT-2 cells and PMCs significantly enhanced proliferation compared with mono-culture of SUIT-2. Original magnification, x200 (left panel), x600 (right panel). (I) Representative images of orientation of collagen fiber using OrientationJ. (J) The percentage of fibers in parallel orientation along the invasive direction $\left(-90\right.$ to $\left.-70^{\circ}, 70-90^{\circ}\right)$ were calculated in five fields of the indicated matrices. Scale bars, $100 \mu \mathrm{m} .{ }^{* *} \mathrm{P}<0.01,{ }^{* * * *} \mathrm{P}<0.001$.

PMCs promote peritoneal dissemination of PCCs in vivo. To investigate the functional role of PMCs on the dissemination of PCCs in vivo, we intraperitoneally injected luciferaseexpressing SUIT-2 cells alone or with PMCs in nude mice and 
A
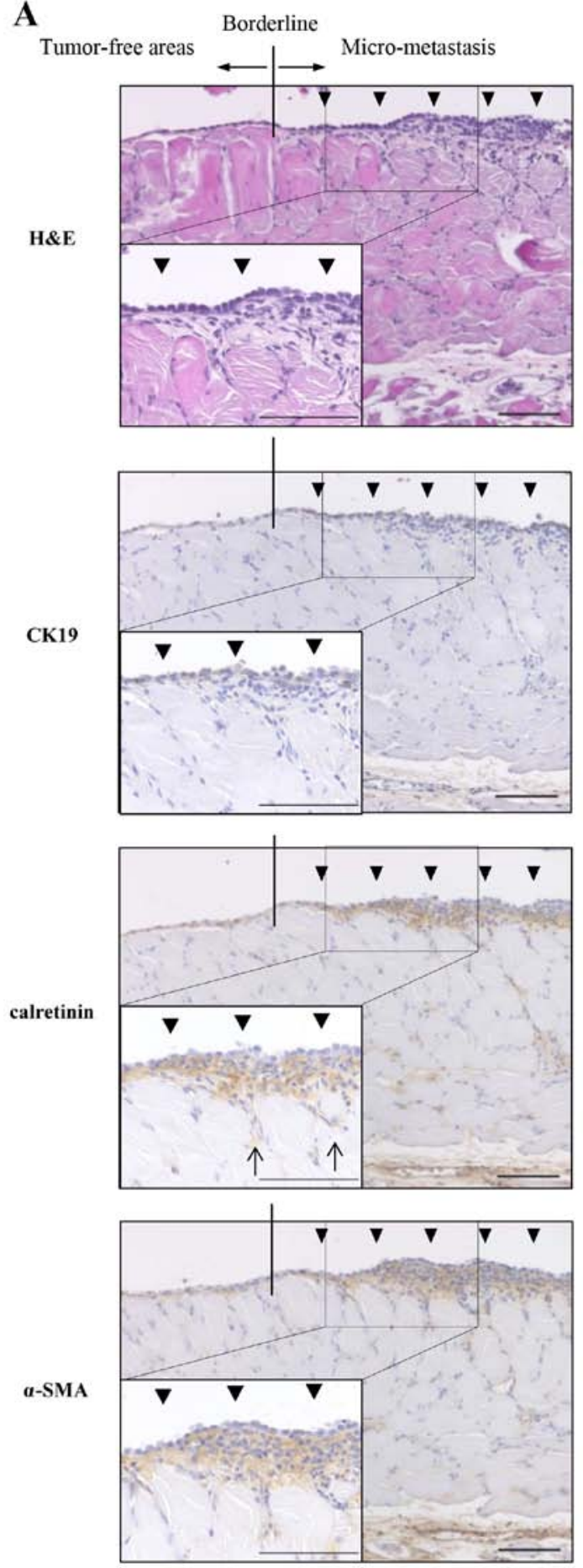

B
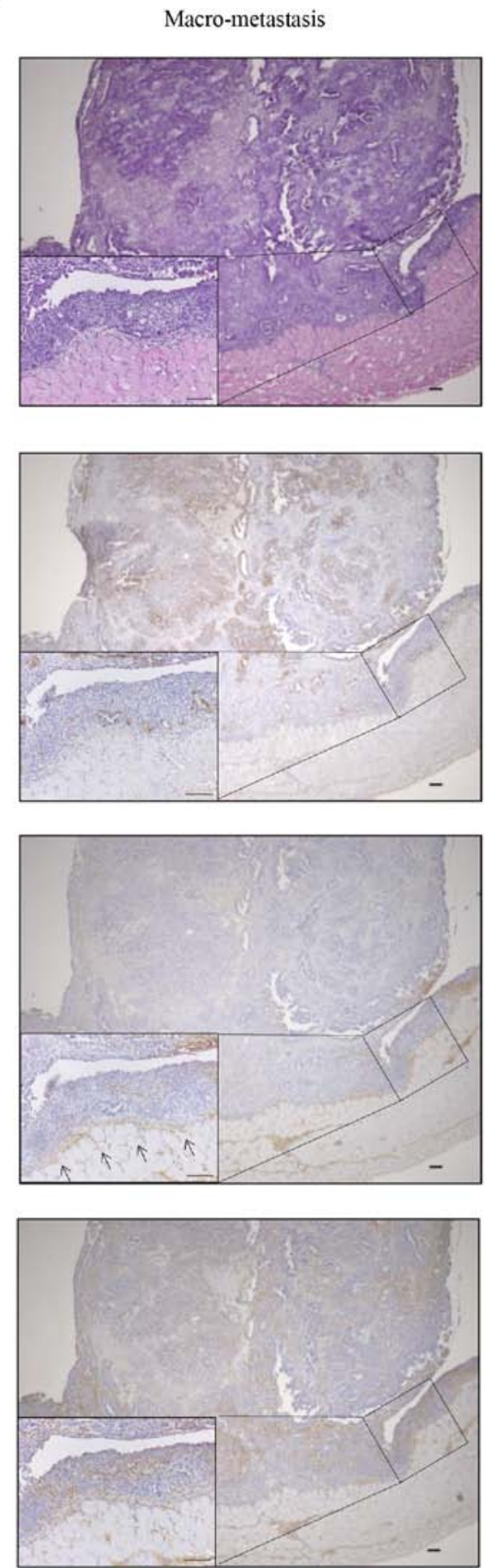

Figure 6. PMCs exist in the invasive front of peritoneal dissemination of pancreatic cancer derived from KPC transgenic mouse. Representative images of H\&E staining and immunohistochemical staining for CK19, calretinin and $\alpha$-SMA in peritoneal dissemination derived from the KPC mouse. (A) The monolayer of PMCs was preserved in tumor-free areas, whereas in micro-peritoneal dissemination, multiple layers of PMCs were observed and a subset of PMCs invaded into the muscle layer. (B) In macro-peritoneal dissemination, PMCs existed in the invasive front and pre-invaded into the muscle layer, whereas $\alpha$-SMApositive and calretinin-negative stromal cells existed in the tumor. The arrowheads indicate cancer cells and the arrow indicate PMCs. Scale bars, $100 \mu \mathrm{m}$.

measured the luciferase luminescence to evaluate the growth of disseminated nodules of SUIT-2 cells. We found that intraperitoneal injection of SUIT-2 cells with PMCs significantly promoted peritoneal dissemination compared with SUIT-2 cells alone (Fig. 7A and B; $\mathrm{P}<0.01$ ). Intraperitoneal injection of SUIT-2 cells with PMCs (Fig. 7C, right panel) yielded a mean $29.3 \pm 14.1$ peritoneal disseminated nodules $>3 \mathrm{~mm}$ compared with $7.0 \pm 4.8$ nodules in mice injected with SUIT-2 cells alone
(Fig. 7C, left panel), a difference that was statistically significant (Fig. 7D; $\mathrm{P}<0.01)$.

\section{Discussion}

In the present study, we found that the tumor-stromal interaction of PCCs and PMCs significantly enhanced their migration and invasiveness and enhanced proliferation and 
A

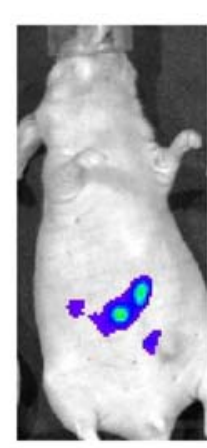

SUIT-2

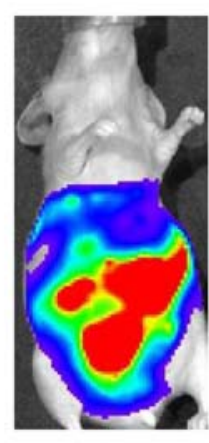

SUIT-2+PMCs

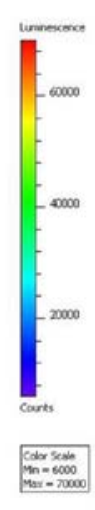

B

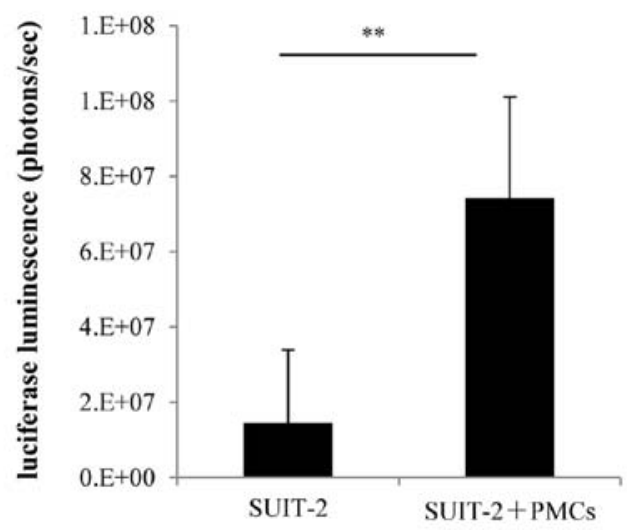

D

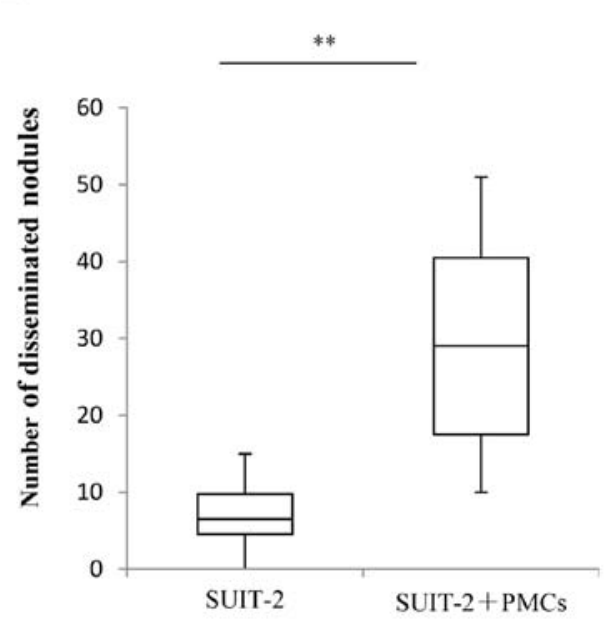

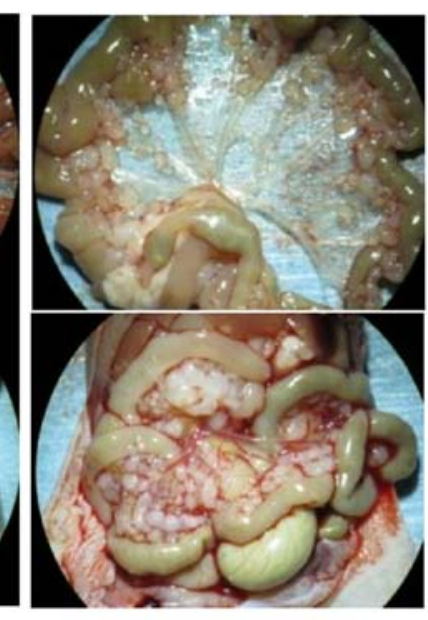

SUIT-2+PMCs

SUIT-2+PMCs
SUIT-2

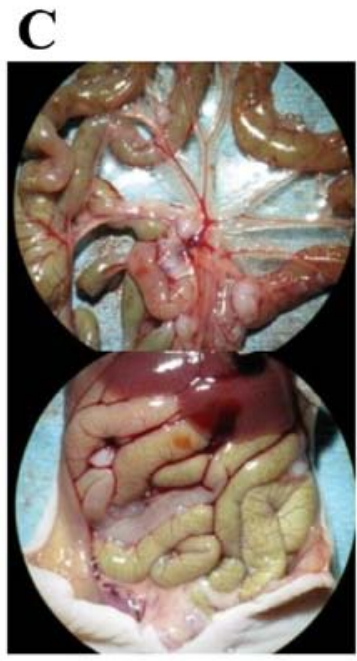

Figure 7. PMCs promote peritoneal dissemination of PCCs in vivo. (A) Representative photograph of IVIS imaging system in nude mice intraperitoneally injected luciferase-expressing SUIT-2 cells alone or with PMCs. (B) Quantification of luciferase luminescence in mice injected luciferase-expressing SUIT-2 cells alone or with PMCs; ${ }^{* *} \mathrm{P}<0.01$. (C) Representative photographs of peritoneal dissemination in nude mice intraperitoneally injected with SUIT-2 cells alone or with PMCs at 21 days. (D) Quantification of disseminated nodules in mice with intraperitoneal injection of SUIT-2 cells with PMCs or with SUIT-2 cells alone; ${ }^{* *} \mathrm{P}<0.01$.

anoikis resistance of PCCs. In the 3D organotypic culture model, we found that co-culture with PCCs and PMCs significantly increased the numbers of cells invading into the collagen gel layer compared with monoculture of PCCs. We also found that PMCs pre-invaded into the collagen gel, remodeled collagen fibers, and increased parallel fiber orientation along the direction of cell invasion. We recently reported that 3D matrices with the parallel fiber architecture derived from pancreatic stellate cells (PSCs) under hypoxia promoted cancer cell motility by inducing directional migration of PCCs (30). These findings suggest that PMCs might enhance the directional invasion of PCCs by increasing parallel fiber orientation besides growth factors associated with the tumor-stromal interaction.

Kasagi et al (31) showed that peritoneal lavage fluid that contained peritoneal collagen type IV and plasma fibronectin facilitated spheroid formation of colon cancer cells. Moreover, Condello et al (32) revealed that spheroid formation of cancer cells under non-adherent conditions served to protect cells from environmental-induced anoikis. We demonstrated that PMCs enhanced proliferation in non-adhered conditions and anoikis resistance of PCCs. These findings suggest that PMCs promote spheroid formation of cancer cells and contribute to survival of cancer cells in the peritoneal fluid.

Next, we demonstrated that the adhesion ability of PCCs to PMCs was significantly decreased compared with Collagen I, whereas the adhesion ability of PCCs to aPMCs was significantly increased compared with PMCs. These findings were similar to previous reports in gastric cancer (33). A previous study also showed that the expression of FN1 in PMCs that was stimulated by ovarian cancer cells promoted the adhesion of cancer cells in ovarian cancer $(33,34)$. In the present study, the expression of FN1 in PMCs with MMT was significantly increased compared with PMCs (Fig. 1B and C). These findings indicate that the expression of FN1 in PMCs with MMT might be involved in promoting the adhesion of PCCs.

We demonstrated that growth factors associated with tumorstromal interaction of PCCs and PMCs mutually enhanced their migration and invasiveness in indirect co-culture. Moreover, aPMCs significantly enhanced the migration and invasiveness of PCCs. In the 3D organotypic culture model of peritoneal dissemination, co-culture of SUIT-2 and PMCs 
significantly increased the number of cells invading into the collagen gel layer compared with monoculture of SUIT-2 cells, which did not invade into the collagen gel layer. The interaction of cancer cells and PMCs enhanced the invasiveness of cancer cells in ovarian (14) and gastric (20) cancer in the 3D organotypic culture model. Moreover Satoyoshi et al (20) revealed that Tks5 activation in PMCs created the invasion front of peritoneal metastasis, which guided invasiveness of cancer cells. In the present study, we revealed that PMCs pre-invaded into the collagen gel, remodeled collagen fibers, and increased parallel fiber orientation along the direction of cell invasion. Conklin et al (35) revealed that the presence of straightened collagen fibers is a predictor of breast cancer survival. Furthermore, we previously reported that $3 \mathrm{D}$ matrices with the parallel fiber architecture derived from PSCs under hypoxia promoted cancer cell motility by inducing directional migration of PCCs (30). These findings suggest that a subset of PMCs enhanced the directional invasion of PCCs by increasing parallel fiber orientation besides growth factors associated with tumor-stromal interaction.

In previous studies, the interaction of cancer cells and PMCs in the murine tissues of peritoneal dissemination was assessed in vivo using intraperitoneal injection of cancer cells into nude mice $(14,20)$. However, these models might not reflect the spontaneous formation of peritoneal dissemination because peritoneal dissemination of these models was artificially created. Therefore, in the present study, we assessed the tissues of peritoneal dissemination that were spontaneously developed in the KPC mouse with pancreatic cancer, which histologically recapitulates the human tumors. Similar to our findings in the 3D organotypic culture model, the monolayer of PMCs was preserved in tumor-free areas, whereas PMCs were present in the invasive front of micro-peritoneal dissemination and proliferated there, and a subset of PMCs invaded into the muscle layer. These findings indicate that PMCs pre-invade, possibly to lead invasiveness of PCCs through the tumorstroma interaction and the matrices remodeling.

We also showed that intraperitoneal injection of PCCs and PMCs significantly promoted peritoneal dissemination compared with PCCs alone in vivo. Similar results were reported in gastric (20) and ovarian cancer (19). In the present study, exogenous PMCs promoted peritoneal dissemination in vivo possibly by enhancing proliferation, anoikis resistance and invasiveness of PCCs.

In conclusion, our results suggest that a subset of PMCs promote the formation of peritoneal dissemination through the tumor-stromal interaction and the matrices remodeling although PMCs were generally thought to play a protective role for peritoneal dissemination. Therapy targeting this specific subset of PMCs may improve the prognosis of patients with pancreatic cancer.

\section{Acknowledgements}

The authors thank E. Manabe, S. Sadatomi and M. Ohmori (Department of Surgery and Oncology, Kyushu University Hospital). The present study was supported in part by a Japan Society for the Promotion of Science Grant-in-Aid for Fellows (no. 16J03962) and Scientific Research (B) and (C) and Scientific Research on Innovative Areas (grant nos. 26293305,
15K10185, 25713050，16K15621，16K10601，16K10600, $16 \mathrm{H} 05417,15 \mathrm{H} 04933,15 \mathrm{~K} 15498$ and $16 \mathrm{H} 05418)$.

\section{References}

1. Siegel R, Ma J, Zou Z and Jemal A: Cancer statistics, 2014. CA Cancer J Clin 64: 9-29, 2014.

2. Thomassen I, Lemmens VE, Nienhuijs SW, Luyer MD, Klaver YL and de Hingh IH: Incidence, prognosis, and possible treatment strategies of peritoneal carcinomatosis of pancreatic origin: A population-based study. Pancreas 42: 72-75, 2013.

3. Sadeghi B, Arvieux C, Glehen O, Beaujard AC, Rivoire M, Baulieux J, Fontaumard E, Brachet A, Caillot JL, Faure JL, et al: Peritoneal carcinomatosis from non-gynecologic malignancies: Results of the EVOCAPE 1 multicentric prospective study. Cancer 88: 358-363, 2000.

4. Neesse A, Michl P, Frese KK, Feig C, Cook N, Jacobetz MA, Lolkema MP, Buchholz M, Olive KP, Gress TM, et al: Stromal biology and therapy in pancreatic cancer. Gut 60: 861-868, 2011.

5. Tan DS, Agarwal R and Kaye SB: Mechanisms of transcoelomic metastasis in ovarian cancer. Lancet Oncol 7: 925-934, 2006.

6. Sugarbaker PH: Peritoneum as the first-line of defense in carcinomatosis. J Surg Oncol 95: 93-96, 2007.

7. Yashiro $\mathrm{M}$ and Hirakawa K: Cancer-stromal interactions in scirrhous gastric carcinoma. Cancer Microenviron 3: 127-135, 2010.

8. Akagawa S, Ohuchida K, Torata N, Hattori M, Eguchi D, Fujiwara K, Kozono S, Cui L, Ikenaga N, Ohtsuka T, et al: Peritoneal myofibroblasts at metastatic foci promote dissemination of pancreatic cancer. Int J Oncol 45: 113-120, 2014.

9. Yáñez-Mó M, Lara-Pezzi E, Selgas R, Ramírez-Huesca M, Domínguez-Jiménez C, Jiménez-Heffernan JA, Aguilera A, Sánchez-Tomero JA, Bajo MA, Alvarez V, et al: Peritoneal dialysis and epithelial-to-mesenchymal transition of mesothelial cells. N Engl J Med 348: 403-413, 2003.

10. Tsukada T, Fushida S, Harada S, Yagi Y, Kinoshita J, Oyama K, Tajima H, Fujita H, Ninomiya I, Fujimura T, et al: The role of human peritoneal mesothelial cells in the fibrosis and progression of gastric cancer. Int J Oncol 41: 476-482, 2012.

11. Epperly MW, Guo H, Gretton JE and Greenberger JS: Bone marrow origin of myofibroblasts in irradiation pulmonary fibrosis. Am J Respir Cell Mol Biol 29: 213-224, 2003.

12. Xu Z, Vonlaufen A, Phillips PA, Fiala-Beer E, Zhang X, Yang L, Biankin AV, Goldstein D, Pirola RC, Wilson JS, et al: Role of pancreatic stellate cells in pancreatic cancer metastasis. Am J Pathol 177: 2585-2596, 2010.

13. Mutsaers SE: The mesothelial cell. Int J Biochem Cell Biol 36: 9-16, 2004.

14. Sandoval P, Jiménez-Heffernan JA, Rynne-Vidal Á, PérezLozano ML, Gilsanz Á, Ruiz-Carpio V, Reyes R, García-Bordas J, Stamatakis K, Dotor J, et al: Carcinoma-associated fibroblasts derive from mesothelial cells via mesothelial-to-mesenchymal transition in peritoneal metastasis. J Pathol 231: 517-531, 2013.

15. Na D, Lv ZD, Liu FN, Xu Y, Jiang CG, Sun Z, Miao ZF, Li F and Xu HM: Transforming growth factor betal produced in autocrine/paracrine manner affects the morphology and function of mesothelial cells and promotes peritoneal carcinomatosis. Int J Mol Med 26: 325-332, 2010.

16. Yamaguchi $\mathrm{H}$ and Sakai R: Direct interaction between carcinoma cells and cancer associated fibroblasts for the regulation of cancer invasion. Cancers (Basel) 7: 2054-2062, 2015.

17. Scherz-Shouval R, Santagata S, Mendillo ML, Sholl LM, Ben-Aharon I, Beck AH, Dias-Santagata D, Koeva M, Stemmer SM, Whitesell L, et al: The reprogramming of tumor stroma by HSF1 is a potent enabler of malignancy. Cell 158: 564-578, 2014.

18. Ksiazek K, Mikula-Pietrasik J, Korybalska K, Dworacki G, Jörres A and Witowski J: Senescent peritoneal mesothelial cells promote ovarian cancer cell adhesion: The role of oxidative stress-induced fibronectin. Am J Pathol 174: 1230-1240, 2009.

19. Mikuła-Pietrasik J, Sosińska P, Kucińska M, Murias M, Maksin K, Malińska A, Ziółkowska A, Piotrowska H, Woźniak A and Książek K: Peritoneal mesothelium promotes the progression of ovarian cancer cells in vitro and in a mice xenograft model in vivo. Cancer Lett 355: 310-315, 2014.

20. Satoyoshi R, Aiba N, Yanagihara K, Yashiro M and Tanaka M: Tks5 activation in mesothelial cells creates invasion front of peritoneal carcinomatosis. Oncogene 34: 3176-3187, 2015. 
21. Sluiter N, de Cuba E, Kwakman R, Kazemier G, Meijer G and Te Velde EA: Adhesion molecules in peritoneal dissemination: Function, prognostic relevance and therapeutic options. Clin Exp Metastasis 33: 401-416, 2016.

22. Kanda M and Kodera Y: Molecular mechanisms of peritoneal dissemination in gastric cancer. World J Gastroenterol 22: 6829-6840, 2016.

23. Yung S, Li FK and Chan TM: Peritoneal mesothelial cell culture and biology. Perit Dial Int 26: 162-173, 2006.

24. Kitayama J, Emoto S, Yamaguchi $\mathrm{H}$, Ishigami $\mathrm{H}$ and Watanabe $\mathrm{T}$ : $\mathrm{CD} 0^{+}$mesothelial-like cells in peritoneal fluid promote peritoneal metastasis by forming a tumor permissive microenvironment. PLoS One 9: e86516, 2014.

25. Whitehead RH and Hughes LE: Tissue culture studies. Br J Cancer 32: 512-518, 1975.

26. Ohuchida K, Mizumoto K, Murakami M, Qian LW, Sato N, Nagai E, Matsumoto K, Nakamura T and Tanaka M: Radiation to stromal fibroblasts increases invasiveness of pancreatic cancer cells through tumor-stromal interactions. Cancer Res 64: 3215-3222, 2004.

27. Hingorani SR, Wang L, Multani AS, Combs C, Deramaudt TB, Hruban RH, Rustgi AK, Chang S and Tuveson DA: Trp53 $3^{R 172 H}$ and $\mathrm{Kras}^{G 12 D}$ cooperate to promote chromosomal instability and widely metastatic pancreatic ductal adenocarcinoma in mice. Cancer Cell 7: 469-483, 2005.

28. Alkhamesi NA, Ziprin P, Pfistermuller K, Peck DH and Darzi AW: ICAM-1 mediated peritoneal carcinomatosis, a target for therapeutic intervention. Clin Exp Metastasis 22: 449-459, 2005.

29. Rezakhaniha R, Agianniotis A, Schrauwen JTC, Griffa A, Sage D, Bouten CV, van de Vosse FN, Unser M and Stergiopulos N: Experimental investigation of collagen waviness and orientation in the arterial adventitia using confocal laser scanning microscopy. Biomech Model Mechanobiol 11: 461-473, 2012.
30. Sada M, Ohuchida K, Horioka K, Okumura T, Moriyama T, Miyasaka Y, Ohtsuka T, Mizumoto K, Oda Y and Nakamura M: Hypoxic stellate cells of pancreatic cancer stroma regulate extracellular matrix fiber organization and cancer cell motility. Cancer Lett 372: 210-218, 2016.

31. Kasagi Y, Harada Y, Morodomi Y, Iwai T, Saito S, Yoshida K, Oki E, Saeki H, Ohgaki K, Sugiyama M, et al: Peritoneal dissemination requires an Sp1-dependent CXCR4/CXCL12 signaling axis and extracellular matrix-directed spheroid formation. Cancer Res 76: 347-357, 2016.

32. Condello S: Morgan C a, Nagdas S, Cao L, Turek J, Hurley TD and Matei D: $\beta$-Catenin-regulated ALDH1A1 is a target in ovarian cancer spheroids. Oncogene 34: 1-12, 2014.

33. Lv Z-D, Na D, Liu F-N, Du ZM, Sun Z, Li Z, Ma XY, Wang ZN and $\mathrm{Xu} \mathrm{HM}$ : Induction of gastric cancer cell adhesion through transforming growth factor-beta1-mediated peritoneal fibrosis. J Exp Clin Cancer Res 29: 139, 2010.

34. Kenny HA, Chiang CY, White EA, Schryver EM, Habis M, Romero IL, Ladanyi A, Penicka CV, George J, Matlin K, et al: Mesothelial cells promote early ovarian cancer metastasis through fibronectin secretion. J Clin Invest 124: 4614-4628, 2014.

35. Conklin MW, Eickhoff JC, Riching KM, Pehlke CA, Eliceiri KW, Provenzano PP, Friedl A and Keely PJ: Aligned collagen is a prognostic signature for survival in human breast carcinoma. Am J Pathol 178: 1221-1232, 2011. 